\title{
SOM FRICATIVO SONORO /3/: MODIFICAÇÕES VOCAIS
}

\author{
Fricative hearing sound /3/: vocal modifications
}

Helena D’Avila (1), Carla Aparecida Cielo ${ }^{(2)}$, Márcia do Amaral Siqueira ${ }^{(3)}$

\begin{abstract}
RESUMO
Objetivo: verificar as modificações vocais após a utilização do fricativo sonoro /3/, em dez mulheres sem alterações vocais/laríngeas. Métodos: foram realizadas medidas acústicas, perceptivo-auditiva, eletroglotografia e auto-avaliação da voz pré e pós-produção da técnica. Resultados: foram estatisticamente significantes: as sensações subjetivas positivas; a maior definição de harmônicos e de formantes, diminuição do ruído, e maior regularidade no traçado. Conclusão: a técnica promove estabilidade vocal, gerando menor esforço fonatório, maior conforto durante a produção vocal e maior projeção vocal no grupo estudado.
\end{abstract}

DESCRITORES: Voz; Fonoterapia; Distúrbios da Voz; Qualidade da Voz

\section{INTRODUÇÃO}

A qualidade vocal é, nos dias atuais, considerada o mais completo atributo de um indivíduo, e sua avaliação fornece indícios sobre os parâmetros físicos, psicológicos, sociais e culturais ${ }^{1}$. Alguns autores levantam questões que podem ser consideradas para avaliar a adequação da voz, sendo possível perceber se as características acústicas são estética e socialmente aceitáveis e se oferecem ao ouvinte inteligibilidade adequada da fala ${ }^{2,3}$.

A voz é considerada um fenômeno multidimensional, exigindo instrumentos multidimensionais para sua avaliação e reabilitação ${ }^{1,3,4}$. O objetivo principal da avaliação da voz é desenvolver um plano de terapia e os secundários são: educar o paciente sobre a produção da voz e identificar técnicas terapêuticas que serão utilizadas no tratamento ${ }^{3,5}$.

A terapia visa ao melhor desempenho da voz do falante, o que nem sempre significa uma voz

(1) Fonoaudióloga; Consultório particular, Dourados, Mato Grosso do Sul; Especialista em Fonoaudiologia pela Universidade Federal de Santa Maria.

(2) Fonoaudióloga; Professora Adjunto do Departamento de Fonoaudiologia da Universidade Federal de Santa Maria, Santa Maria, RS; Doutora em Linguística Aplicada pela Pontifícia Universidade Católica do Rio Grande do Sul.

(3) Fonoaudióloga do Centro de Referência em Saúde do Trabalhador, CEREST, Santa Maria, RS; Colaboradora da Universidade Federal de Santa Maria, UFSM, Santa Maria, RS; Mestre em Distúrbios da Comunicação Humana pela Universidade Federal de Santa Maria.

Conflito de interesses: inexistente "normal", porém adequada para a comunicação daquela pessoa. Os elementos gerais para a terapia incluem aconselhamento sobre higiene, exercícios ou treinamento vocais e integração do comportamento recentemente obtido. A principal meta do treinamento vocal é modificar a produção da voz por meio de novos ajustes e memórias musculares ${ }^{3,6}$.

Com esse objetivo, são utilizadas várias técnicas de terapia, cuja seleção depende de uma série de fatores, sendo um deles o conhecimento e a familiaridade com o exercício vocal, além da eficácia comprovada da técnica selecionada. As técnicas vocais estão incluídas em todos os procedimentos fonoaudiológicos, a saber: na prevenção, na reabilitação, e no aperfeiçoamento vocal. Estudos que comprovem a eficácia de uma determinada técnica vocal são raros, havendo grande heterogeneidade entre o que já se sabe sobre o mecanismo vocal e a aplicação clínica desse conhecimento.

A categoria das consoantes fricativas do português brasileiro é composta por seis fonemas: /f/, /v/, /s/, /z/, / / /, /3/, os mais fracos do português, com valores entre 15 e 25 dBNA. Quanto mais próxima dos lábios situa-se a fonte friccional, mais agudo é o fone; os fonemas $/ \mathrm{J} /$, e /3/ são os mais graves, com zona entre 2500 e $6000 \mathrm{~Hz}$; os fonemas /s/ e /z/ são os mais agudos, com zona acima de $4500 \mathrm{~Hz}$, chegando a $8000 \mathrm{~Hz}$. Os fonemas fricativos /z/, /v/, e /3/ são considerados sonoros por apresentarem vibração de pregas vocais durante a emissão, ao contrário dos fonemas fricativos surdos /s/, /f/, e / / / 
que não apresentam vibração de pregas vocais durante sua emissão e são, em média, $40 \mathrm{~ms}$ mais longos do que os sonoros ${ }^{1,7-11}$.

A utilização de exercícios vocais que contenham o som fricativo sonoro pode auxiliar a melhora da hipernasalidade e a adução glótica ${ }^{12}$, bem como da hipertensão fonatória, da incoordenação pneumofônica, dos ataques vocais bruscos, da direção do fluxo aéreo para o ambiente, dos tempos máximos de fonação, do apoio respiratório, e do controle da intensidade ${ }^{13,14}$.

A produção prolongada de um som fricativo parece causar elevação da posição vertical da laringe no pescoço, diminuição da atividade dos músculos da laringe, e contato aumentado entre as pregas vocais ${ }^{15}$. Durante o som fricativo, a atividade dos músculos da laringe diminui, possivelmente como consequência da ressonância retroflexa causada pela resistência supraglótica, permanecendo estabilizada na produção da vogal após o som fricativo. Este fato leva a uma fonação mais econômica, caracterizada pela possibilidade de adquirir a mesma produção vocal com menos esforço, mais economia e eficiência vocal ${ }^{16,17}$.

Apesar de alguns estudos ${ }^{1-17}$, ainda há carência de documentação dos efeitos das técnicas vocais terapêuticas, sendo uma importante lacuna nas pesquisas na área de voz, notando-se a necessidade de trabalhos científicos nessa área, com enfoque na reabilitação.

Com base no exposto, a presente pesquisa tem como objetivo verificar as modificações vocais ocasionadas pela produção do som de apoio de fricativo sonoro $/ 3 /$, em indivíduos adultos do sexo feminino sem queixa vocal e sem patologia laríngea.

\section{MÉTODOS}

Trata-se de pesquisa de campo, exploratória, transversal, prospectiva, quanti-qualitativa, envolvendo 10 adultos jovens brasileiros do sexo feminino.

Os critérios de inclusão para o estudo foram: concordar em participar da pesquisa; ter habilidade para executar o fricativo sonoro /3/; ser do sexo feminino; e encontrar-se na $1^{\circ}$ metade da fase de adulto jovem que, geralmente, tem início no final da adolescência (cerca de 20 anos de idade) e término aos 40 anos ${ }^{17,18}$. Nessa faixa etária, o aparelho fonador ainda não sofreu a influência das alterações hormonais e estruturais do envelhecimento, como também não sofre mais as alterações do período da muda vocal.

Os critérios de exclusão foram: apresentar hábitos de etilismo e ou tabagismo; alterações alérgicas, faríngeas, orais, nasais, pulmonares, digestivas e endócrinas; alterações vocais, laríngeas, e história pregressa de disfonia; conhecimento da técnica vocal estudada; alterações auditivas, pois poderiam modificar o feedback vocal, comprometendo a qualidade vocal; e alterações do sistema estomatognático que pudessem interferir na articulação da fala, comprometendo a voz e a execução do fricativo sonoro /3/.

Para a seleção do grupo de estudo, por meio da aplicação dos critérios de inclusão e de exclusão, os sujeitos foram questionados oralmente sobre seus dados de identificação, idade, sexo, histórico de saúde, e hábitos vocais ${ }^{1,12}$; foram submetidos à avaliação otorrinolaringológica, com objetivo de descartar alterações e queixas laríngeas; realizaram triagem auditiva ${ }^{19}$ individualmente e em sala silenciosa, com o objetivo de verificação dos limiares auditivos aéreos por meio de audiômetro digital (Fonix, FA-12, Tipo I) e fones auriculares (TDH-39P, Telephonics); e realizaram a avaliação do sistema estomatognático e de suas funções com o objetivo de investigar as condições estruturais e funcionais dos órgãos fonoarticulatórios.

Após a seleção dos sujeitos, a coleta dos dados referentes à voz foi realizada no período vespertino, com o intuito de evitar que o repouso vocal da noite interferisse no desempenho na coleta da amostra de voz.

Durante a coleta das amostras de voz, o sujeito permaneceu em pé com os braços estendidos ao longo do corpo, o microfone profissional (Yoga $\circledR$ unidirecional - DM 585-600 ohm) foi posicionado à frente da boca, adaptado a um pedestal, para manter constantes a distância de $15 \mathrm{~cm}$ e ângulo de $90^{\circ} \mathrm{em}$ relação à boca; na eletroglotografia, o glotógrafo foi colocado nas alas da cartilagem tireóide, na altura das pregas vocais por meio de dois eletrodos de superfície, com o pescoço previamente higienizado com solução alcoólica. As gravações foram feitas em sala acusticamente tratada ${ }^{1,20}$.

Foram realizadas gravações da emissão da vogal sustentada $/ \varepsilon: /$, em tom e intensidade habituais de fala, durante pelo menos três segundos 12,14,21, sem esforço, pré (Figura 1) e pósexecução do fricativo sonoro /3/ (Figura 2).

Dessas amostras de voz, foram extraídos dados referentes às avaliações vocais perceptivo-auditiva, acústica da fonte glótica, eletroglotográfica, e espectrográfica, antes e após o fricativo sonoro /3/, e foi também realizada a auto-avaliação vocal após a execução da técnica.

Para a avaliação vocal perceptivo-auditiva, foram analisados os parâmetros de ressonância (oral, equilibrada, laríngea, faríngea, laringofaríngea, hipernasal, hiponasal, denasal), tipo de voz (rouca, soprosa, áspera, fluida e comprimida), 


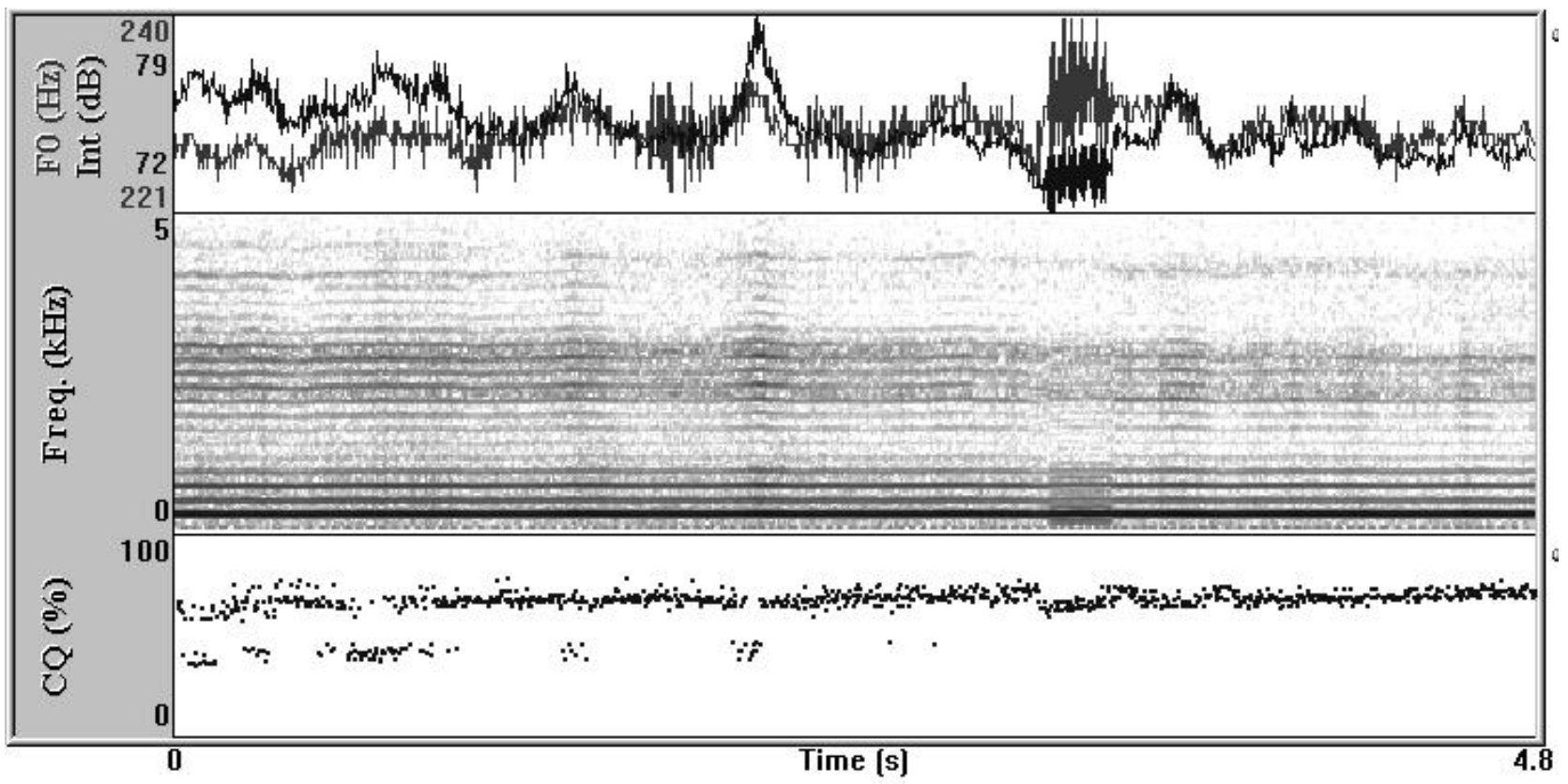

Figura 1 - EX PRÉ-TÉCNICA (pouca definição de harmônicos, presença de ruído em todo o espectro, substituição de harmônicos por ruído nas freqüências altas)

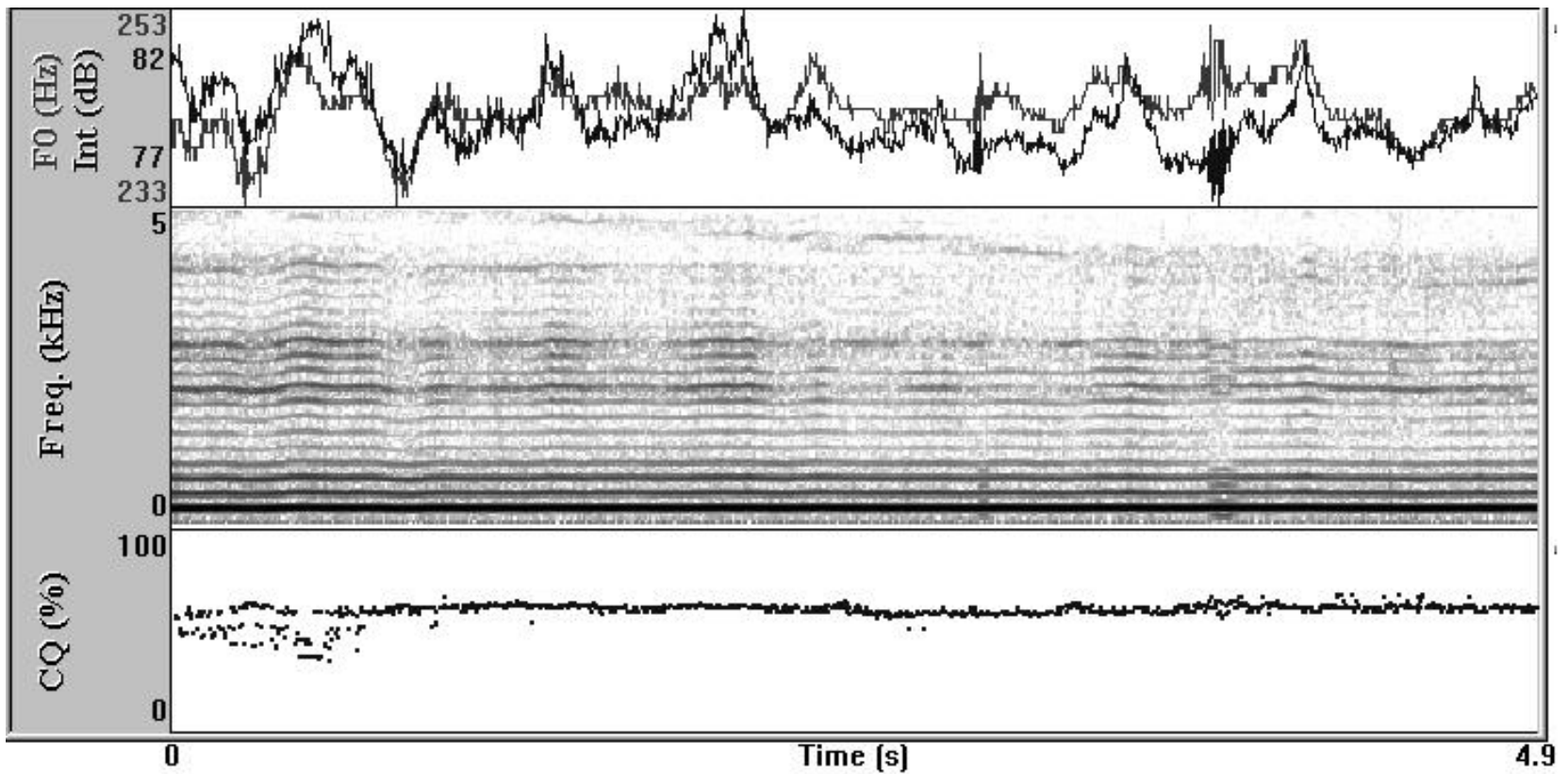

Figura 2 - EX PÓS-TÉCNICA (maior definição de harmônicos, diminuição de ruído em todo o espectro, diminuição de ruído nas freqüências altas)

graus (normal, leve, moderado, e intenso) e qualidade da emissão (instabilidade e flutuações, quebras de sonoridade, bitonalidade, variações na altura e na intensidade, uso de ar de reserva expiratória, variações na frequência e intensidade 1,12,22. Quatro fonoaudiólogas realizaram a avaliação independentemente umas das outras, sem conhecimento entre a emissão pré e pósrealização da técnica, considerando-se os dados predominantes das suas análises nas categorias de melhora, piora, e inalterado ou igual.

A extração das medidas acústicas da fonte vocal e a eletroglotografia foram realizadas simultaneamente pelo programa computadorizado Software 
Doctor Speech, version 4.0, Voice Assessment da Tiger Eletronics $\AA$, e glotógrafo EG80 da F-J Eletronics $\AA$, com a calibração do ruído, do sinal vocal e da freqüência fundamental na faixa feminina, conforme o grupo de estudo.

Para as medidas de fonte glótica, foram extraídos: frequência fundamental média (f0), jitter, shimmer, energia de ruído glótico (ERG), e proporção harmônico-ruído (PHR) ${ }^{1,12,23}$.

$\mathrm{Na}$ eletroglotografia, foram analisados: frequência fundamental média na eletroglotografia (f0 EGG), jitter na eletroglotografia, shimmer na eletroglotografia, energia de ruído glótico na eletroglotografia (ERG EGG), proporção harmônico-ruído na eletroglotografia (PHR EGG), quociente de contato $(\mathrm{QC})$, e perturbação do quociente de contato (PQC) ${ }^{1,12,24}$.

A análise acústica espectrográfica de banda larga e de banda estreita foi realizada considerando-se a definição de harmônicos (h), presença de ruído, definição de formantes $(F)$, e regularidade do traçado ${ }^{1,12}$, julgadas como melhor, pior ou inalterada, considerando-se o pré e o pós-técnica. A análise espectrográfica foi efetuada por três fonoaudiólogas, com experiência na área de voz, independentemente uma da outra, considerando-se os dados predominantes das suas análises nas categorias de melhora, piora, e inalterado ou igual.

Para a execução da técnica vocal, solicitouse ao sujeito que emitisse o fricativo sonoro /3/ ${ }^{13}$, em tempo máximo de fonação, em duas séries de quinze vezes. Cada série teve intervalo de um minuto de repouso vocal absoluto ${ }^{25}$. A técnica foi executada com o indivíduo sentado de forma confortável, em tom e intensidade habituais de fala, sem deslocamento cervical e com os pés apoiados no chão e coluna ereta ${ }^{1}$.

A auto-avaliação vocal foi realizada apenas ao término das coletas de voz, pós-execução da técnica vocal. O sujeito foi indagado sobre o que percebeu de modificação em sua voz, tanto auditivamente, quanto proprioceptivamente e as respostas foram categorizadas em: melhora, piora, e inalterado ou igual. Esta pesquisa foi aprovada pelo Comitê de Ética em Pesquisa da instituição de origem ( $\left.n^{\circ} 164\right)$ e os sujeitos leram e assinaram o Termo de Consentimento Livre e Esclarecido como recomenda a norma 196/96 da Comissão Nacional de Ética em Pesquisa - CONEP/1996.

Ao final da coleta de dados, os mesmos foram tabulados, calculando-se a significância estatística entre as diferenças das medidas da análise acústica da fonte glótica e da eletroglotografia, e calculandose os percentuais das avaliações perceptivo-auditiva e espectrográfica, e da auto-avaliação vocal.

Para a análise estatística dos dados, foi realizado o teste Paramétrico t-pareado devido aos elementos receberem um tipo de tratamento ou nenhum tratamento, para posteriormente serem comparados aos pares provenientes de duas amostras relacionadas com nível de significância 5\% e nível de confiança de $95 \%$. Para a análise dos dados que foram analisados subjetivamente, foi usado o teste não-paramétrico dos Sinais com nível de significância $5 \%$ e nível de confiança de $95 \%$, $z$ tabelado $=1,64$, probabilidades $p=0,5$ e $q=0,5$.

\section{RESULTADOS}

Os valores descritivos obtidos para o Teste Paramétrico t-pareado em cada variável da análise acústica da fonte glótica podem ser observados na Tabela 1.

Os valores do teste estatístico paramétrico t-pareado obtidos para cada variável da análise acústica da fonte glótica podem ser observados na Tabela 2.

Como o valor de $p$ encontrado para todas as variáveis foi maior do que 0,05 , aceita-se $\mathrm{HO}$, ou seja, o resultado não foi considerado significante estatisticamente (Tabela 3).

Como o valor de $p$ encontrado para todas as variáveis foi maior do que 0,05 , aceita-se $\mathrm{HO}$, ou seja, o resultado não foi considerado significante estatisticamente (Tabela 4).

Como o valor de $p$ encontrado para todas as variáveis foi maior do que 0,05 , aceita-se $\mathrm{HO}$, ou seja, o resultado não foi considerado significante estatisticamente (Tabela 5).

O resultado obtido após a aplicação do teste não-paramétrico dos Sinais evidenciou o valor do $z$ tabelado $=1,90$ para a espectrografia, de 1,67 para o tipo de voz e de 2,33 para a auto-análise vocal. Portanto, o resultado do teste não-paramétrico dos Sinais para estas variáveis foi estatisticamente significante.

Quanto à auto-avaliação vocal, nos resultados positivos, foram citadas as seguintes características: "voz limpa e clara, com maior facilidade de produção, menos tremida, mais regular, forçando menos na emissão, e conseguindo manter mais a voz". Nos resultados negativos, mencionou-se "voz mais tremida". 
Tabela 1 - Valores descritivos obtidos para o Teste Paramétrico t-pareado em cada variável da análise acústica da fonte glótica

\begin{tabular}{lccccccc}
\hline \multirow{2}{*}{ Variável } & N & \multicolumn{2}{c}{ Média } & \multicolumn{2}{c}{ Desvio-padrão } & \multicolumn{2}{c}{ Limites de confiança } \\
\cline { 2 - 8 } & & Antes & Depois & Antes & Depois & $\begin{array}{c}\text { Limite } \\
\text { inferior }\end{array}$ & $\begin{array}{c}\text { Limite } \\
\text { superior }\end{array}$ \\
\hline f0 média (Hz) & 10 & 208,192 & 215,649 & 26,968 & 35,512 & $-19,97$ & 5,060 \\
Jitter (\%) & 10 & 0,446 & 0,573 & 0,002 & 0,005 & $-0,500$ & 0,300 \\
Shimmer (\%) & 10 & 3,693 & 3,905 & 0,011 & 0,018 & $-1,600$ & 1,100 \\
ERG (dB) & 10 & $-7,706$ & $-7,875$ & 4,059 & 3,862 & $-1,213$ & 1,550 \\
PHR (dB) & 10 & 23,423 & 23,287 & 3,204 & 3,977 & $-2,265$ & 2,537 \\
\hline
\end{tabular}

Medidas descritivas: Média - Média Aritmética; Desvio Padrão - estimativa de variância; Limites de confiança - valores extremos de cada classe ; ERG - ruído glótico; PHR - proporção harmônico-ruído

Tabela 2 - Valores do teste estatístico paramétrico t- pareado obtidos para cada variável da análise acústica da fonte glótica

\begin{tabular}{ccccccccccc}
\hline \multirow{2}{*}{ Sujeito } & \multicolumn{2}{c}{ f0 Média (Hz) EGG } & \multicolumn{2}{c}{ Jitter (\%) EGG } & \multicolumn{2}{c}{ Shimmer (\%) EGG } & \multicolumn{2}{c}{ ERG (dB) EGG } & \multicolumn{2}{c}{ PHR (dB) EGG } \\
\cline { 2 - 10 } & Antes & Depois & Antes & Depois & Antes & Depois & Antes & Depois & Antes & Depois \\
\hline 1 & 229,93 & 0,93 & 0,40 & 0,25 & 5,12 & 3,25 & $-6,68$ & $-9,23$ & 20,60 & 25,85 \\
2 & 191,50 & 0,67 & 0,59 & 0,37 & 4,13 & 3,76 & $-3,56$ & $-2,51$ & 22,84 & 21,77 \\
3 & 191,09 & 0,36 & 0,28 & 0,19 & 3,02 & 3,06 & $-5,25$ & $-7,17$ & 24,01 & 24,47 \\
4 & 251,99 & 0,40 & 1,99 & 0,91 & 3,59 & 7,52 & $-2,42$ & $-2,75$ & 22,26 & 18,09 \\
5 & 239,46 & 0,29 & 0,31 & 0,22 & 2,82 & 2,73 & $-7,60$ & $-6,98$ & 27,59 & 26,59 \\
6 & 218,24 & 0,22 & 0,30 & 0,19 & 2,39 & 2,97 & $-9,71$ & $-10,37$ & 27,75 & 24,04 \\
7 & 188,55 & 0,36 & 0,46 & 0,27 & 1,90 & 2,48 & $-16,34$ & $-12,83$ & 27,58 & 27,86 \\
8 & 164,48 & 0,51 & 0,49 & 0,31 & 5,25 & 2,86 & $-9,41$ & $-11,92$ & 22,04 & 25,51 \\
9 & 212,27 & 0,39 & 0,68 & 0,43 & 4,66 & 7,27 & $-5,36$ & $-3,71$ & 19,24 & 15,06 \\
10 & 194,38 & 0,33 & 0,23 & 0,17 & 4,05 & 3,15 & $-10,73$ & $-11,28$ & 20,32 & 23,63 \\
Média & 208,18 & 0,44 & 0,57 & 0,33 & 3,69 & 3,90 & $-7,70$ & $-7,87$ & 23,42 & 23,28 \\
\hline p & 0,210945 & 0,487648 & 0,732939 & 0,788279 & & 0,900883 \\
\hline$t$ tabelado & 2,262159 & 2,262158887 & 2,262158887 & 2,262158887 & 2,262158887 \\
\hline
\end{tabular}

*significância estatística; fo Média - freqüência fundamental média; ERG - ruído glótico; PHR - proporção harmônico-ruído, Jitter normal até $0,5 \%$ e Shimmer normal até $3,0 \%$

Tabela 3 - Medidas descritivas do teste estatístico paramétrico t-pareado realizado para cada variável da eletroglotografia

\begin{tabular}{|c|c|c|c|c|c|c|c|}
\hline \multirow[b]{2}{*}{ Variável } & \multirow[b]{2}{*}{$\mathbf{N}$} & \multicolumn{2}{|c|}{ Média } & \multicolumn{2}{|c|}{ Desvio-Padrão } & \multicolumn{2}{|c|}{ Limites de confiança } \\
\hline & & Antes & Depois & Antes & Depois & $\begin{array}{l}\text { Limite } \\
\text { inferior }\end{array}$ & $\begin{array}{l}\text { Limite } \\
\text { superior }\end{array}$ \\
\hline f0 media $(\mathrm{Hz})$ & 10 & 208,188 & 215,644 & 26,965 & 35,515 & $-19,978$ & 5,066 \\
\hline Jitter (\%) & 10 & 0,282 & 0,331 & 0,0976 & 0,2199 & $-0,2$ & 0,1 \\
\hline Shimmer (\%) & 10 & 2,234 & 2,467 & 0,6085 & 1,1204 & $-0,9$ & 0,5 \\
\hline $\mathrm{ERG}(\mathrm{dB})$ & 10 & $-20,327$ & $-20,174$ & 3,154 & 3,911 & $-2,509$ & 2,202 \\
\hline PHR (dB) & 10 & 23,416 & 23,28 & 3,195 & 3,955 & $-2,246$ & 2,518 \\
\hline CQ (\%) & 10 & 52,345 & 52,57 & 13,146 & 10,753 & $-6,097$ & 5,647 \\
\hline CQP (\%) & 10 & 4,075 & 4,05 & 3,763 & 4,990 & $-4,422$ & 4,472 \\
\hline
\end{tabular}

Medidas descritivas: Média - Média Aritmética; Desvio Padrão - estimativa de variância; Limites de confiança - valores extremos de cada classe ; f0 Média - freqüência fundamental média; ERG - ruído glótico; PHR - proporção harmônico-ruído; $C Q$ - quociente de contato , CQP- perturbação do quociente de contato, Jitter normal até $0,5 \%$ e Shimmer normal até 3,0\% 
Tabela 4 - Valores do teste estatístico paramétrico t- pareado obtidos para cada variável da eletroglotografia

\begin{tabular}{|c|c|c|c|c|c|c|c|c|c|c|c|c|c|c|}
\hline \multirow[t]{2}{*}{ Sujeito } & \multicolumn{2}{|c|}{ fo Média $(\mathrm{Hz})$ EGG } & \multicolumn{2}{|c|}{ Jitter (\%) EGG } & \multicolumn{2}{|c|}{$\begin{array}{c}\text { Shimmer }(\%) \\
\text { EGG }\end{array}$} & \multicolumn{2}{|c|}{$E R G(d B) E G G$} & \multicolumn{2}{|c|}{ PHR (dB) EGG } & \multicolumn{2}{|c|}{ QC EGG } & \multicolumn{2}{|c|}{ PQC EGG } \\
\hline & Antes & Depois & Antes & Depois & Antes & Depois & Antes & Depois & Antes & Depois & Antes & Depois & Antes & Depois \\
\hline 1 & 229,93 & 243,61 & 0,50 & 0,25 & 2,79 & 1,93 & $-17,72$ & $-22,93$ & 20,71 & 25,86 & 65,44 & 59,31 & 4,83 & 2,03 \\
\hline 2 & 191,50 & 175,99 & 0,39 & 0,37 & 2,43 & 2,43 & $-19,59$ & $-18,47$ & 22,79 & 21,75 & 59,29 & 61,37 & 2,12 & 1,94 \\
\hline 3 & 191,09 & 206,82 & 0,24 & 0,19 & 1,82 & 1,83 & $-20,45$ & $-21,29$ & 24,00 & 24,46 & 47,70 & 38,16 & 5,73 & 4,31 \\
\hline 4 & 251,99 & 239,03 & 0,27 & 0,91 & 2,23 & 4,27 & $-19,19$ & $-14,87$ & 22,24 & 18,16 & 57,13 & 59,12 & 1,45 & 3,93 \\
\hline 5 & 239,46 & 287,04 & 0,22 & 0,22 & 1,56 & 2,13 & $-24,55$ & $-23,68$ & 27,56 & 26,57 & 62,53 & 65,24 & 1,17 & 0,97 \\
\hline 6 & 218,24 & 222,66 & 0,16 & 0,19 & 1,60 & 2,08 & $-24,57$ & $-20,67$ & 27,75 & 23,95 & 58,45 & 56,79 & 0,68 & 0,87 \\
\hline 7 & 188,55 & 198,08 & 0,24 & 0,27 & 1,44 & 1,52 & $-24,56$ & $-24,79$ & 27,56 & 27,85 & 52,66 & 61,18 & 3,89 & 0,79 \\
\hline 8 & 164,48 & 166,01 & 0,30 & 0,31 & 3,08 & 1,74 & $-19,09$ & $-22,49$ & 22,05 & 25,48 & 34,60 & 40,03 & 13,55 & 4,22 \\
\hline 9 & 212,27 & 221,92 & 0,28 & 0,43 & 3,00 & 4,79 & $-16,33$ & $-12,52$ & 19,22 & 15,09 & 60,91 & 46,97 & 2,37 & 17,66 \\
\hline 10 & 194,38 & 195,28 & 0,22 & 0,17 & 2,39 & 1,95 & $-17,22$ & $-20,52$ & 20,28 & 23,63 & 24,74 & 37,53 & 4,96 & 3,78 \\
\hline Média & 208,18 & 215,64 & 0,28 & 0,33 & 2,23 & 2,46 & $-20,32$ & $-20,17$ & 23,41 & 23,28 & 52,34 & 52,57 & 4,07 & 4,05 \\
\hline$p$ & \multicolumn{2}{|c|}{0,210945} & \multicolumn{2}{|c|}{0,518193} & \multicolumn{2}{|c|}{0,50456} & \multicolumn{2}{|c|}{0,886388} & \multicolumn{2}{|c|}{0,900092} & \multicolumn{2}{|c|}{0,932826} & \multicolumn{2}{|c|}{0,990133} \\
\hline$t$ tabelado & \multicolumn{2}{|c|}{2,262159} & \multicolumn{2}{|c|}{2,262159} & \multicolumn{2}{|c|}{2,262159} & \multicolumn{2}{|c|}{2,262159} & \multicolumn{2}{|c|}{2,262159} & \multicolumn{2}{|c|}{2,262159} & \multicolumn{2}{|c|}{2,262159} \\
\hline
\end{tabular}

* significância estatística; Nível de significância 5\%; Nível de confiança 95\%; f0 Média - freqüência fundamental média; ERG - ruído glótico na eletroglotografia; PHR - proporção harmônico-ruído na eletroglotografia; $C Q$ - quociente de contato na eletroglotografia , CQP- perturbação do quociente de contato na eletroglotografia, Jitter normal até 0,5\% e Shimmer normal até 3,0\%

Tabela 5 - Valores do teste estatístico Não-paramétrico dos Sinais obtidos para os resultados da espectrografia e da análise vocal perceptivo-auditiva, antes e depois da técnica e da auto-análise da voz depois da técnica

\begin{tabular}{|c|c|c|c|c|c|c|c|c|c|c|c|c|c|c|c|}
\hline \multirow{3}{*}{$\begin{array}{c}\text { Percentual } \\
(n=10)\end{array}$} & \multirow{2}{*}{\multicolumn{3}{|c|}{ Espectrografia }} & \multicolumn{9}{|c|}{ Análise Vocal Perceptivo-auditiva } & \multirow{2}{*}{\multicolumn{3}{|c|}{ Auto-análise Vocal }} \\
\hline & & & & \multicolumn{3}{|c|}{ Tipo de Voz } & \multicolumn{3}{|c|}{ Ressonância } & \multicolumn{3}{|c|}{ Qualidade da Emissão } & & & \\
\hline & Melhor & Pior & Igual & Melhor & Pior & Igual & Melhor & Pior & Igual & Melhor & Pior & Igual & Melhor & Pior & Igual \\
\hline & $80 \%$ & $20 \%$ & $0 \%$ & $70 \%$ & $20 \%$ & $10 \%$ & $60 \%$ & $10 \%$ & $30 \%$ & $30 \%$ & $20 \%$ & $50 \%$ & $80 \%$ & $10 \%$ & $10 \%$ \\
\hline$z$ & & $1,90^{*}$ & & & $1,67^{\star}$ & & & 1,22 & & & 0,45 & & & $2,33^{*}$ & \\
\hline
\end{tabular}

* significância estatística

\section{DISCUSSÃO}

As Tabelas de 1 a 4 mostram que os resultados encontrados na comparação pré e pós-produção da técnica vocal estudada não foram considerados significantes para cada variável analisada. Os resultados encontrados na eletroglotografia são semelhantes aos encontrados na análise acústica da fonte glótica e, desta forma, serão comentados conjuntamente.

A literatura evidencia que a frequência fundamental corresponde ao número de ciclos glóticos por segundo, refletindo as características biomecânicas das pregas vocais em sua interação com a pressão subglótica 1,12,14. A frequência fundamental é típica para os sexos, a voz feminina normal apresenta maior frequência fundamental, podendo variar de $204 \mathrm{~Hz}$ a $234 \mathrm{~Hz}{ }^{1,12,19,22}$. Nesta pesquisa, pôde-se perceber que a produção do som fricativo sonoro não influenciou significativamente a frequência fundamental média, já que a variação entre a pré e a pós-execução da técnica mostrou-se sem significância estatística, porém os achados encontram-se dentro dos padrões de referência para o sexo feminino.

Explicando esse pequeno aumento da f0 média depois da produção da técnica vocal de fricativo sonoro $/ 3 /$, apesar de não significante estatisticamente, pode-se afirmar que, na produção dessa técnica, a língua fica com a sua porção anterior e corpo elevados acima da sua posição neutra, elevando a laringe e resultando no encurtamento do trato vocal, com uma subseqüente elevação de todas as frequências dos formantes ${ }^{1}$.

Verificou-se um alto pico de frequência no espectro para a produção de / $/ \mathrm{e} / 3 / \mathrm{em}$ torno de $4500 \mathrm{~Hz}$ em mulheres ${ }^{26}$, fato que pode justificar a discreta elevação da frequência fundamental após a realização da técnica no presente trabalho. A frequência é controlada pela extensão das pregas vocais, sua tensão, pressão aérea subglótica e amplitude de vibração ${ }^{27}$. No momento em que ocorre a elevação da laringe, a tensão das pregas vocais aumenta, resultando em um som mais agudo, o que se encontrou neste estudo. Uma laringe alta contribui para a amplificação de harmônicos mais agudos, interferindo também nesse 
processo o posicionamento dos órgãos fonoarticulatórios que influencia a qualidade vocal final 1,12,27, sendo que o arcabouço laríngeo, o osso hióide e a base da língua estão envolvidos diretamente na produção vocal, visto que a posição articulatória linguopalatal do fonema fricativo sonoro //3/ impõe essas características.

Os resultados deste estudo, embora não significantes, também vão ao encontro da afirmação ${ }^{15}$ de que a produção prolongada de um som fricativo parece causar uma elevação da posição vertical da laringe, e conseqüentemente, da frequência fundamental.

No entanto, os resultados deste trabalho são discordantes do estudo ${ }^{28}$ que sugere que uma constrição no trato vocal poderia ocasionar a diminuição da freqüência fundamental. Esse autor está provavelmente se referindo ao som fricativo sonoro anterior ou línguo-dental $/ \mathrm{v} /$, em que não ocorre elevação do dorso da língua.

De acordo com a literatura, o jitter indica as variações de altura em torno da frequência fundamental, representando a instabilidade vocal. Também indica alteração em função das condições fonatórias, intensidade, frequência de emissão e do decréscimo do controle sobre o sistema fonatório ${ }^{1}$. A variável jitter, ou seja, a perturbação na altura, também não sofreu influência da técnica vocal de fricativo sonoro, sendo que o valor encontrado na análise acústica, antes da produção da técnica vocal, mostrou-se dentro do esperado para o sexo, e o valor encontrado após a produção da técnica mostrou-se elevado em relação ao esperado para o sexo, porém, essa elevação em relação ao padrão de normalidade, não foi significante estatisticamente, como também não o foi a diferença entre o pré e o pós-técnica.

No jitter-EGG, os valores se encontraram dentro do esperado para o sexo feminino, sendo que a diferença pré e pós-técnica não foi significante estatisticamente.

Na literatura, o shimmer é definido como uma variação em intensidade (amplitude) em torno da frequência fundamental, sendo uma medida baseada no pico da amplitude em cada ciclo fonatório. É uma medida que serve para quantificar a instabilidade do sinal vocal ${ }^{1}$.

Durante a produção dos sons fricativos ocorre um aumento acentuado na superfície de atrito do ar, gerando instabilidade em função das condições fonatórias. Assim, quando se faz uso da produção de sons fricativos sonoros, ocorre aumento da pressão de ar supraglótica, consequência da constrição do trato vocal, afetando a vibração das pregas vocais. Em consequência disso, ocorrem variações em torno da intensidade vocal ${ }^{15,27}$.
A variável shimmer, no presente trabalho, também não sofreu influência da técnica vocal de fricativo sonoro /3/, pois a variação entre a pré e a pós-execução da técnica vocal foi muito pequena, não mostrando significância estatística. $O$ valor encontrado na análise da voz pré e pós-técnica vocal mostrou-se fora do padrão de normalidade esperado para o sexo, entretanto essa variação também não foi estatisticamente significante. Para o shimmer-EGG, os valores obtidos se encontraram dentro do padrão de normalidade esperado para o sexo e não evidenciaram diferença significante entre o pré e o pós-técnica.

$\mathrm{Na}$ variável ERG, variante da PHR, que se refere ao ruído da onda sonora ao nível da glote, obteve-se resultados distintos entre a análise da fonte glótica e a eletroglotografia. Na ERG, ocorreu discreta redução após a realização da técnica, estando os valores de ERG pré e pós-execução da técnica acima do padrão de normalidade esperado para o sexo, entretanto, tais variações não foram consideradas significantes estatisticamente. $\mathrm{Na}$ ERG-EGG, houve o fenômeno inverso, ocorrendo discreto aumento após a execução da técnica, embora seus valores pós-execução tenham se apresentado dentro do padrão de normalidade esperado para o sexo. A técnica vocal de fricativo sonoro, não causou influência nessas variáveis, já que os resultados obtidos não foram significantes estatisticamente.

De acordo com a literatura, a PHR contrasta o sinal regular das pregas vocais com o sinal irregular das mesmas e do trato vocal, oferecendo um índice que relaciona o componente harmônico com o componente de ruído da onda acústica ${ }^{1}$. Nesta pesquisa, percebeu-se que a variação da PHR, a variação da PHR-EGG, e a variação entre ambas não foram estatisticamente significantes, mostrando que o som fricativo sonoro não influenciou a PHR, embora tenha havido discreta diminuição em ambas, podendo sugerir diminuição do ruído glótico após o fricativo /3/.

$\mathrm{Na}$ literatura, O CQ representa o quociente de contato entre as pregas vocais. É uma medida do grau de aproximação das pregas vocais durante a fonação, que fornece dados importantes sobre a área de contato das mesmas durante a vibração, revelando o grau de fechamento glótico, ou seja, reflete a atividade em nível laríngeo ${ }^{1,29}$.

$\mathrm{Na}$ presente pesquisa, pôde-se perceber que o som fricativo sonoro /3/ não influenciou o fechamento glótico, visto que a variação entre os valores da pré e da pós-execução da técnica não foi estatisticamente significante, sendo que os valores obtidos nos dois momentos se encontraram dentro do padrão de normalidade esperado para o sexo, 
embora tenha havido discreto aumento, sugerindo maior quociente de contato ou maior aproximação de pregas vocais. Alguns autores ${ }^{12,13,22}$ consideram a técnica de fricativos como sendo facilitadora da emissão, propiciando um melhor equilíbrio funcional da produção vocal, ou seja, normotensora, justificando o discreto aumento, embora não significativo do $C Q$, após a execução do fricativo sonoro.

A literatura evidencia que $P Q C$ representa uma variação ciclo a ciclo do $C Q$. A PQC também apresentou variação estatisticamente não significante, embora tenha ocorrido discreta diminuição após a técnica, reforçando os dados da literatura de que a técnica do fricativo sonoro proporciona maior equilíbrio fonatório ${ }^{13}$. Os achados pré e pós-execução da técnica estão acima do padrão de normalidade esperado, contudo, essa variação não foi estatisticamente significante.

Os espectrogramas refletem as propriedades da fonte de som (características vibratórias das pregas vocais) e do ressoador (trato vocal). Quando observada a efetividade da técnica de som fricativo sonoro /3/ em relação à análise espectrográfica, após a aplicação do teste Não-paramétrico dos Sinais, evidenciou-se que o resultado obtido para a avaliação espectrográfica foi estatisticamente significante, confirmando a melhora observada em $80 \%$ dos sujeitos após a realização da técnica vocal estudada. O resultado da análise espectrográfica mostrou maior definição de harmônicos e de formantes, com conseqüente regularidade do traçado espectrográfico e menor presença de ruído após a técnica.

Os harmônicos são indicativos da qualidade vocal, quanto mais harmônicos evidentes no espectrograma, melhor o padrão de produção sonora analisada, evidenciando o papel do sistema de ressonância da voz ${ }^{1}$. Tais resultados são concordantes com, a afirmação de que a utilização do som fricativo sonoro pode auxiliar a melhora da hipernasalidade ${ }^{27}$.

A produção prolongada de um som fricativo parece levar a uma fonação mais econômica, caracterizada pela possibilidade de adquirir a mesma produção vocal com menos esforço ${ }^{15,17}$, o que poderia ser associado ao equilíbrio ressonantal proporcionado pela técnica. O efeito esperado para quem utiliza fonemas fricativos sonoros é uma dissociação entre o aumento de intensidade e esforço vocal, favorecendo o direcionamento adequado do fluxo aéreo sonorizado para o sistema de ressonância, e promovendo maior projeção da voz ${ }^{13}$.
$\mathrm{Na}$ avaliação do tipo de voz, a aplicação do teste Não-paramétrico dos Sinais evidenciou melhora estatisticamente significante em $70 \%$ dos sujeitos após a realização da técnica vocal estudada. Considerando-se os resultados estatisticamente significantes obtidos na espectrografia, observa-se concordância com a melhora apontada pelos fonoaudiólogos-juízes quanto ao tipo de voz.

$\mathrm{Na}$ avaliação perceptivo-auditiva da ressonância, a realização do teste Não-paramétrico dos Sinais mostrou não mudança estatisticamente significante, mas a maioria dos sujeitos obteve melhora nesse parâmetro, concordando com os achados obtidos na espectrografia e com a literatura que mostra o fricativo sonoro como promotor da ressonância e da projeção vocais.

Para a avaliação perceptivo-auditiva da qualidade da emissão, o resultado obtido no teste Nãoparamétrico dos Sinais foi estatisticamente significante. $O$ resultado dessa análise baseou-se em instabilidade e flutuações, sendo que a maioria dos sujeitos permaneceu com a qualidade da emissão inalterada. De acordo com a análise acústica da fonte glótica e a eletroglotografia, não houve alterações significativas de jitter, shimmer, podendo justificar a manutenção do padrão de qualidade vocal.

De forma geral, com ênfase na melhora significativa do tipo de voz, e considerando-se os parâmetros de ressonância e de qualidade vocal, pode-se verificar que a literatura aponta o fricativo sonoro como uma técnica normotensora, que melhora a adução glótica, melhora a ressonância, aumenta a estabilidade vocal, diminui os desvios acústicos, reduz 0 atrito entre as pregas vocais, proporciona diminuição da atividade muscular laríngea, em casos de hipertensão muscular, levando a uma fonação mais econômica, mostrando a possibilidade de alcançar a mesma produção com menos esforço laríngeo, e reduz o ataque vocal brusco, suavizando o início da sonorização 12,14,15,17,27,30. Esses benefícios podem ser relacionados à melhor qualidade vocal, com menor ruído e maior estabilidade e à melhor ressonância, refletindo-se numa melhora do tipo vocal.

O resultado obtido após a aplicação do teste Não-paramétrico dos Sinais para a auto-avaliação vocal foi estatisticamente significante, confirmando o resultado positivo observado em $80 \%$ dos sujeitos após a realização da técnica vocal estudada. Com essa análise, percebeu-se que as sensações auditivas e proprioceptivas do paciente são pistas importantes que podem acrescentar dados à avaliação feita pelo fonoaudiólogo. Mudanças sutis são percebidas e sentidas pelo paciente. 
A linha de tratamento sintomática ${ }^{1}$ expõe, como foco, a modificação direta dos sintomas, podendo oferecer resultados vocais imediatos e até mesmo extraordinários, indicando que, quando a técnica vocal promove mudanças perceptíveis para o paciente, e seguramente ele sofre interferências da propriocepção, isso pode ser mais motivador para o tratamento, uma vez que, percebendo resultados imediatos, o paciente tende à adesão ao tratamento.

É importante ressaltar que análises acústicas devem ser complementares e não substitutas das avaliações clínicas subjetivas, pois mudanças numéricas em qualquer parâmetro vocal não refletem necessariamente as sutilezas da produção da voz percebidas pelos ouvintes. Desta forma, com base nos resultados desta pesquisa, percebeuse que a técnica promove a estabilidade vocal, gerando menor esforço fonatório, maior conforto durante a produção vocal e maior projeção vocal no grupo estudado.

\section{CONCLUSÃO}

A técnica fonoterapêutica de som de apoio fricativo sonoro /3/ promoveu, com significância estatística no grupo estudado, sensação subjetiva dos sujeitos de voz mais limpa e clara, com maior facilidade de produção, menos tremida, mais regular, forçando menos a emissão e conseguindo manter mais a voz; maior definição de harmônicos e de formantes, menor presença de ruído, e maior regularidade no traçado espectrográfico; e melhora no tipo de voz. Ainda, para a maioria dos sujeitos, apesar de não significante estatisticamente, houve melhora na ressonância.

Pôde-se concluir que a técnica de fricativo sonoro /3/ revelou maior influência na ressonância do trato vocal do que na fonte glótica, havendo interrelação entre os sinais espectrográficos, perceptivo-auditivos, e proprioceptivos.

\begin{abstract}
Purpose: verify the vocal modifications that occurred after the utilization of the fricative hearing sound $13 /$ in ten adult women that had no vocal laryngeal alterations. Methods: they passed through a vocal and acoustic evaluation as well as hearing perception, and then they auto-evaluated their voices before and after the technique. Results: statistically significant, namely: positive subjective feelings; spectrograms showing more definition for the harmonic and formants, noise reduction, and more regularity on the trace. Conclusion: the technique produced less effort in phonation, more comfort during the vocal production and a more vocal production in the studied group.
\end{abstract}

KEYWORDS: Voice; Speech Therapy; Voice Disorders; Voice Quality

\section{REFERÊNCIAS}

1. Behlau M. Voz: o livro do especialista. vol. 1. Rio de Janeiro: Revinter; 2001.

2. Corazza VR, Silva VFC, Queija DS, Dedivitis RA, Barros APB. Correlação entre os achados estroboscópicos, perceptivo-auditivos e acústicos em adultos sem queixa vocal. Rev Bras Otorrinolaringol. 2004; 70:45-52.

3. Krischke S, Weigewlt S, Hoppe U, Kollner V, Klotz M, Eysholdt U, et al. Quality of live in disphonic patients. J Voice. 2005; 19(1):132-7.

4. Yu P, Ouaknine M, Revis J, Giovanni A. Objective voice analysis for disphonic patients: a multiparametric protocol including acoustic and aerodynamic measurements. J Voice. 2001; 15(4):529-42.
5. Behrman A. Common practices of voice therapists in the evaluation of patients. $\mathrm{J}$ Voice. 2005; 19(3):454-69.

6. Speyer R, Wieneke GH, Wijck-Warnaar I, Dejonckere $\mathrm{PH}$. Effects of voice therapy on the voice range profiles of dysphonic patients. J Voice. 2003; 17(4):544-56.

7. Cielo CA, Casarin MT. Sons fricativos surdos. Rev CEFAC. 2008; 10(3):352-8.

8. Villanueva $P$, Lizana $M L$, Huber $H$, Morán $D$, Fernández MA, Palomino HM. Modificaciones en la articulación de fones en pacientes com aparato ortodóntico fijo lingual. Rev CEFAC. 2007; 9(4):483-89.

9. Matos HM. La articulación del habla em indivíduos com hendiduras labiopalatinas corregidas: estúdio de dos casos. Rev CEFAC. 2006; 8(2):186-97. 
10. Leite AF, Silva SB, Britto ATBO, Ninno CQMSD. Caracterização do ceceio em pacientes de um centro clínico de fonoaudiologia. Rev Soc Bras Fonoaudiol. 2008; 13(1):30-6.

11. Moura SRS, Cielo CA, Nogaro A. Crianças bilíngues alemão/português: dessonorizações na escrita em português. Rev Fono Atual. 2005; 34:28-46.

12. Pinho SMR. Fundamentos em fonoaudiologia: tratando os distúrbios da voz. Rio de Janeiro: Guanabara Koogan; 2003.

13. Behlau M. Voz: o livro do especialista. Vol. 2. Rio de Janeiro: Revinter; 2005.

14. Rossi DC, Munhoz DF, Nogueira CR, Oliveira $\mathrm{CM}$, Britto ATBO. Relação do pico de fluxo expiratório com o tempo de fonação em pacientes asmáticos. Rev CEFAC. 2006; 8(4):509-17.

15. Laukkanen AM, Lindoholm P, Vilkman E, Haataja $\mathrm{K}$; Alku PA. Physiological and acoustic study on voiced bilabial fricative /B/ as a vocal exercise. $J$ Voice. 1996; 10(1):67-77.

16. Cielo CA, Siqueira MA, D’Ávila $H$. Efeitos da técnica fonoterapêutica de fricativo sonoro /Z/ na voz: análise de um caso. Rev Soc Bras Fonoaudiol. 2005; 10(4):232-5.

17. Sampaio M, Oliveira G, Behlau M. Investigação de efeitos imediatos de dois exercícios de trato vocal semi-ocluído. Pró-Fono Rev de Atualização Científica. 2008; 20(4): 261-6.

18. Susan G, Hiss KT, Stuart A. Effect of age, gender, and repeated measures on intraoral air pressure in normal adults. J Voice. 2001; 15(2):159-64.

19. Xue SA, Hao JG. Normative standards for vocal tract dimensionsby race as measured by acoustic pharyngometry. J Voice. 2006; 20(3):391-400.

20. Chen H, Hsiao T, Hsiao L, Chung Y, Chiang $\mathrm{S}$. Outcome of resonant voice therapy for female teachers with voice disorders: perceptual, physiological, acoustic, aerodynamic, and functional measurements. J Voice. 2006; 21(4): 415-25.

21. Bhuta T, Patrick L, Garnett JD. Perceptual evaluation of voice quality and its correlation. $J$ Voice. 2004; 18(3):299-4.

22. Pinho SMR, Pontes P. Músculos intrínsecos da laringe e dinâmica vocal. Vol.1. Rio de Janeiro: Revinter; 2008.

23. Felippe ACN, Grillo MHMM, Grechi TH. Normatização de medidas acústicas para vozes normais. Rev Soc Bras Otorrinolaringol. 2006; 72(5):659-64.

24. Kandogan $T$, Seifert $E$. Influence of aging and sex on voice parameters in patients with unilateral vocal cord paralysis. Laryngoscope. 2005; 115:655-60.

25. Saxon KG, Schneider CM. Vocal exercise physiology. California: Singular Publishing Group; 1995.

26. Fox RA, Nissen SL. Sex-related acoustic changes in voiceless English fricatives. J Speech Lang Hear Res, 2005; 48(5):753-65.

27. Pinho SMR. Fisiologia da fonação. In: Ferreira LP, Befi-Lopes DM, Limongi SCO. Tratado de fonoaudiologia. São Paulo: Roca; 2004. p. 3-10.

28. Zemlin WR. Princípios de anatomia e fisiologia em fonoaudiologia. Porto Alegre: Artes Médicas Sul; 2000.

29. Dromey C, Stathopoulos ET, Sapienza CM. Glottal airflow and electroglottographic measures of function at multiple intensities. J Voice. 1992; 6(1):44-54.

30. Simberg S, Sala E, Tuomainen J, Sellman J, Ronnemaa A. The effectiveness of group therapy for students with mild voice disorders: a controlled clinical trial. J Voice. 2006; 20(1):97-109.

DOI: 10.1590/S1516-18462010005000104

RECEBIDO EM: 15/04/2009

ACEITO EM: 03/01/2010

Endereço para correspondência:

Carla Aparecida Cielo

Rua Cel. Niederauer, 917/407

Santa Maria - RS

CEP: 97015-121

E-mail: fonoaud@terra.com.br 